\title{
THE EFFECTS OF AREA CONTRACTION ON SHOCK WAVE STRENGTH AND PEAK PRESSURE IN SHOCK TUBE
}

\author{
A. M. Mohsen, M. Z. Yusoff, A. Al-Falahi and N. H. Shuaib \\ Centre for Advanced Computational Engineering, College of Engineering \\ Universiti Tenaga Nasional, UNITEN \\ Jalan IKRAM-UNITEN \\ 43009 Kajang, Selangor, Darul Ehsan, Malaysia \\ E-mail: zamri@uniten.edu.my
}

\begin{abstract}
This paper presents an experimental investigation into the effects of area contraction on shock wave strength and peak pressure in a shock tube. The shock tube is an important component of the short duration, high speed fluid flow test facility, available at the Universiti Tenaga Nasional (UNITEN), Malaysia. The area contraction was facilitated by positioning a bush adjacent to the primary diaphragm section, which separates the driver and driven sections. Experimental measurements were performed with and without the presence of the bush, at various diaphragm pressure ratios, which is the ratio of air pressure between the driver (high pressure) and driven (low pressure) sections. The instantaneous static pressure variations were measured at two locations close to the driven tube end wall, using high sensitivity pressure sensors, which allow the shock wave strength, shock wave speed and peak pressure to be analysed. The results reveal that the area contraction significantly reduces the shock wave strength, shock wave speed and peak pressure. At a diaphragm pressure ratio of 10 , the shock wave strength decreases by $18 \%$, the peak pressure decreases by $30 \%$ and the shock wave speed decreases by $8 \%$.
\end{abstract}

Keywords: Shock tube, shock wave, area contraction.

\section{INTRODUCTION}

Shock wave is the phenomenon of a sudden drop of pressure in a very narrow region within a medium. The sudden pressure drop is accompanied by a sudden rise in temperature and density of that medium. There has been much research done in order to generate and investigate this phenomenon. One of the common methods of generating a shock wave is by rushing a gas at high pressure into another gas at a lower pressure by the sudden rupture of a thin aluminium diaphragm in transient high speed flow test facilities (Wintenberger, 2002). The aluminium diaphragm separates the gas at high pressure from the one at the lower pressure. The section that contains the high pressure gas is called the driver section and the driven section contains the lower pressure gas. Shock wave is widely used as a means to produce high-speed flows in short duration, high speed fluid flow test facilities.

High speed fluid flow facilities, such as shock tunnels, shock tubes and gun tunnels are widely used in many applications. For example, IISc hypersonic shock tunnel HST2 was used to measure the aerodynamic forces on a triangular plate that flies 
at a Mach number of 5.75 (Sahoo et al., 2007). Another important application of shock tunnels is to obtain the conditions of supersonic combustion. Aso et al. (2005) used a shock tunnel to investigate the supersonic combustion phenomena and observed that the reflected shock wave heated the air to a stagnation temperature of $2800 \mathrm{~K}$ and the static temperature of the test section was $1550 \mathrm{~K}$. This temperature is sufficient to generate the supersonic combustion with self-ignition. In addition, there have been many different methods used to develop and improve the performance of high speed fluid flow test facilities and to obtain higher values of shock Mach number (Briassulis et al., 1996; Amir et al., 2008). Lu et al. (2009) developed a new technique to improve the performance of these facilities. This technique used a computer-based controller to avoid any overshoot in the stagnation pressure when it is required to bring rapidly a supersonic wind tunnel to its operating condition. Increasing the diaphragm pressure ratio and raising the speed of sound in the driver gas are very effective methods in obtaining higher Mach numbers (Amir et al., 2007). Furthermore, the use of different gas combinations is another effective method of obtaining higher Mach numbers. For example, light gases, such as Helium or Hydrogen are used in the driver section and heavy gases, such as $\mathrm{CO}_{2}$ or air are used in the driven section (Amir et al., 2008, 2009). Some studies have shown that an increase in the initial temperature ratio of the driven/driver gases also leads to an increase in the Mach number (Amir et al., 2008). However, Kaneko et al. (2005) showed that the scale of the facility also affects the performance because larger facilities have higher nozzle supply pressures and therefore, longer steady state times.

Although, many works have investigated high speed flows in shock tubes, to the best of the author's knowledge, there has been nothing published in the literature that describes the effect of area contraction on shock wave strength, shock wave speed and peak pressure, which partly describes the performance of the high speed flow facilities. In many short duration, high speed flow facilities, a small area contraction in the form of a bush at the diaphragm, is normally used to facilitate the diaphragm rupturing process. The present work involved an experimental investigation on the effects of the area contraction on shock wave strength and peak pressure in the shock tube, which is an important component of the short duration, high speed fluid flow test facility, available at the Universiti Tenaga Nasional. Experimental measurements were performed with and without the presence of the bush at various diaphragm pressure ratios. The instantaneous static pressure variations were measured at two locations close to the driven tube end wall, using high sensitivity pressure sensors, which allow the shock wave strength, shock wave speed and peak pressure to be analysed.

\section{OVERVIEW OF THE FACILITY}

The two main sections of the facility are the driver and the driven sections, separated by a thin aluminium diaphragm. These sections have a cylindrical shape with a $50 \mathrm{~mm}$ inner diameter and $90 \mathrm{~mm}$ outer diameter. Further details of the facility are described below and shown in Figure 1. 


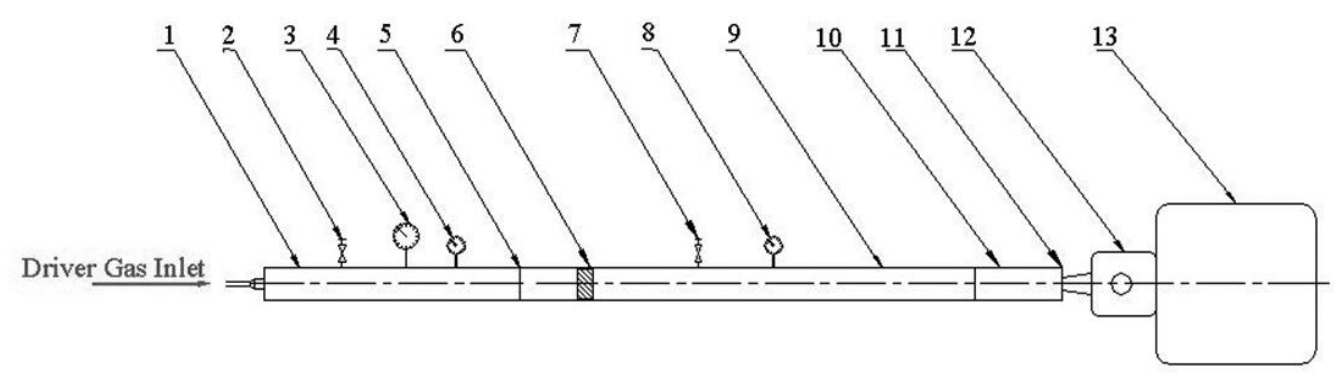

Figure 1. Schematic of the short duration high speed flow test facility at Universiti Tenaga Nasional (UNITEN).

1. Driver section (compression chamber) - The section is a $2.5 \mathrm{~m}$ long SAE-4135 alloy steel pipe. It contains the high pressure driver gas, which can be Air, Helium, Hydrogen or other light gases.

2. Discharge valve - The valve is used to discharge the driver section after each run.

3. Pressure gauge - The pressure gauge is used to measure the pressure inside the driver section. This section is also provided with a static pressure transducer to record the exact value of the driver pressure, $\mathrm{P} 4$ at which the diaphragm ruptures.

4. Vacuum pump - The vacuum pump is used to evacuate air from the driver section when another gas is used as the driver gas (e.g., Helium or Hydrogen).

5. The primary diaphragm - The primary diaphragm is a thin aluminium membrane used to isolate the low-pressure driven gas from the high pressure driver gas. When this membrane ruptured, the compression process is initiated.

6. Piston compression section - When the facility is used as a gun tunnel, a piston is placed in the driven section adjacent to the primary diaphragm. When the diaphragm ruptures, the piston is propelled through the driven tube, compressing the gas ahead of it.

7. Discharge valve - The valve is used to discharge the driven section after each run.

8. Vacuum gauge - The vacuum gauge is used to set the pressure inside the driven section to the desired value (vacuum values) below atmospheric pressure.

9. Driven section (shock tube) - A $3.5 \mathrm{~m}$ long non-machined cold forged uniform tube of alloy steel. It is filled with the driven gas, such as (air, nitrogen or carbon dioxide).

10. Driven section extension - The shock tube extension is $0.5 \mathrm{~m}$ long and has the same internal diameter as shock tube. It is to this section that the pressure transducers and thermocouples are attached.

11. The secondary diaphragm and nozzle section - A light plastic diaphragm to separate the low pressure test gas inside the driven section from the test section and dump tank, which are initially set to a vacuum prior to the run. The main purpose of the second bursting system is to relieve pressurised air rapidly through the convergent-divergent nozzle once the desired pressure has been reached. This will convert subsonic flow to supersonic flow.

12. Test section - This section expands the high temperature test gas through a nozzle to the correct high enthalpy conditions needed to simulate hypersonic 
flow. A range of Mach numbers is available by changing the diameter of the throat insert.

13. Vacuum vessel (dump tank) - This section is to be evacuated to about 0.1 $\mathrm{mmHg}$ pressure before running. Prior to a run, the driven section, test section and dump tank are all evacuated to a low-pressure value.

\section{DIAPHRAGM SECTION}

The diaphragm section consists of two flanges that are designed to hold the thin aluminium diaphragm and connect the driver and driven sections. Four alloy steel bolts of M20 and two O-ring seals are used to join the two flanges and prevent any leaks. The inner diameters of both driver and driven sections are $50 \mathrm{~mm}$. The diaphragm section is shown in Figure 2.

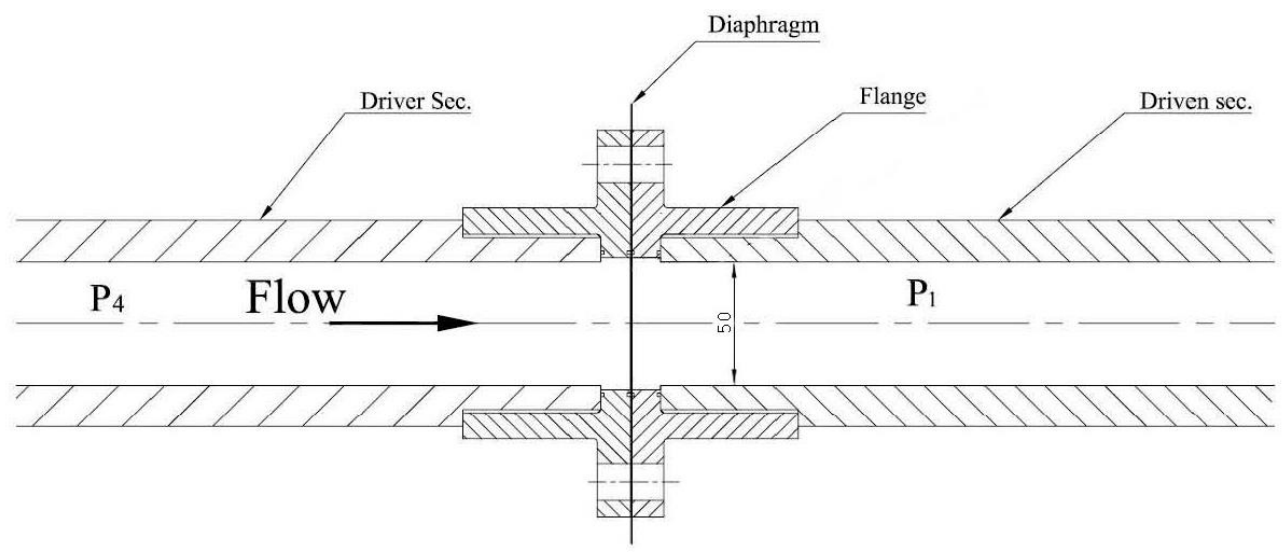

Figure 2. The diaphragm section.
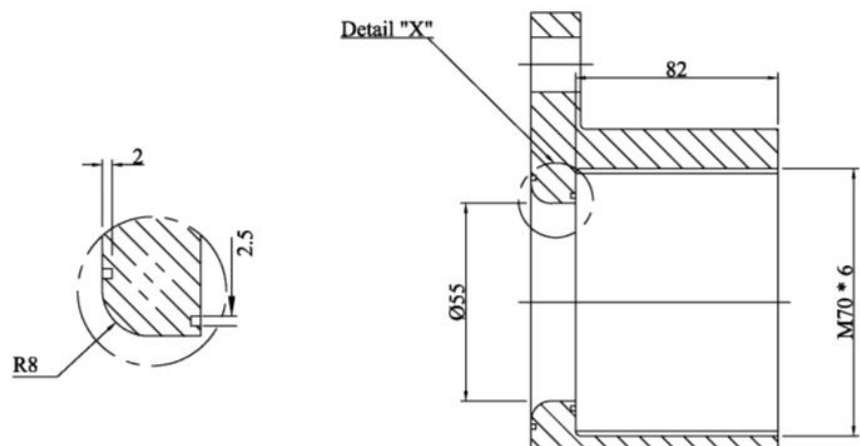

Detail "X"

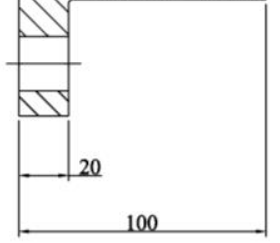

Figure 3. The driven section flange. 
To insert or replace the diaphragm in the diaphragm section, the two flanges have to be separated by loosening the four bolts and the driver section has to be moved away from the driven section. A one-inch turnbuckle is designed to roll the driver section away from the driven section and to bring it back together after the diaphragm is inserted. The internal edge of the driven section side flange has been rounded with a radius of $8 \mathrm{~mm}$ to facilitate the diaphragm rupture process and avoid any shear. This flange is shown in Figure 3. In order to obtain the area contraction in the facility a removable bush has been designed and is placed adjacent to the diaphragm, as shown in Figure 4.
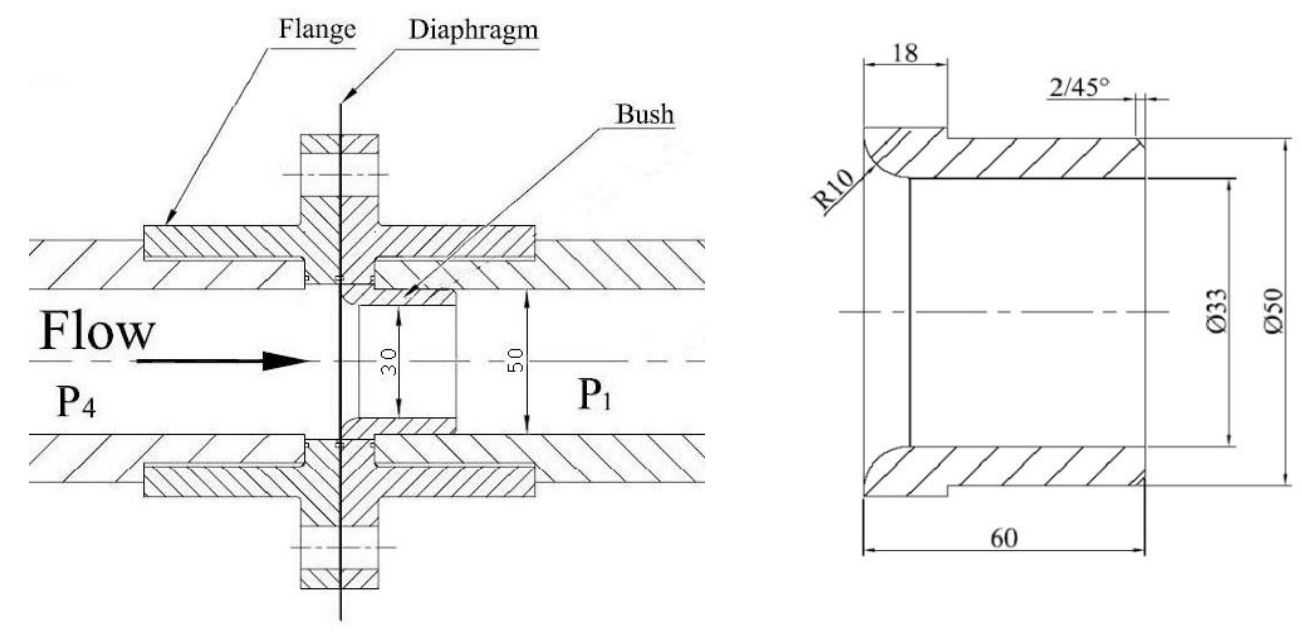

Figure 4. The bush in the driven section adjacent to the diaphragm.

\section{EXPERIMENTAL MEASUREMENTS}

The short duration high speed flow test facility at UNITEN is designed such that it can be operated in four different modes, namely: shock tube, shock tunnel, free piston compressor and gun tunnel. The present work performed the experimental measurements using the shock tube mode. These experimental tests used different operating conditions to investigate the parameters of interest. The initial set of experimental measurements had no area contraction in the facility. In these experiments, a gas combination of air/air as driver/driven gases with three different pressure ratios, $\mathrm{P}_{4} / \mathrm{P}_{1}=10,15$, and 20 were used to obtain the sudden burst of the diaphragm and to generate the shock wave. The driven section pressures were $100 \mathrm{kPa}$ for all cases. The same set of experiments were then repeated with area contraction in the facility (i.e., with the presence of the bush) and the results were compared and analysed to address the effects of the area contraction on the performance of the facility.

In order to measure the instantaneous pressure history in the driven section, two piezo-electric pressure transducers (PCB Piezotronics Inc., model 111A24) with two signal conditioners; one for each sensor (PCB Piezotronics Inc., model 482A21), are mounted at the end of the driven section and separated by an axial distance of $342 \mathrm{~mm}$. The position of the first pressure transducer is station 1 and the second position is station 2, which is located $40 \mathrm{~mm}$ from the end wall of the driven section, as shown in Figure 5. 


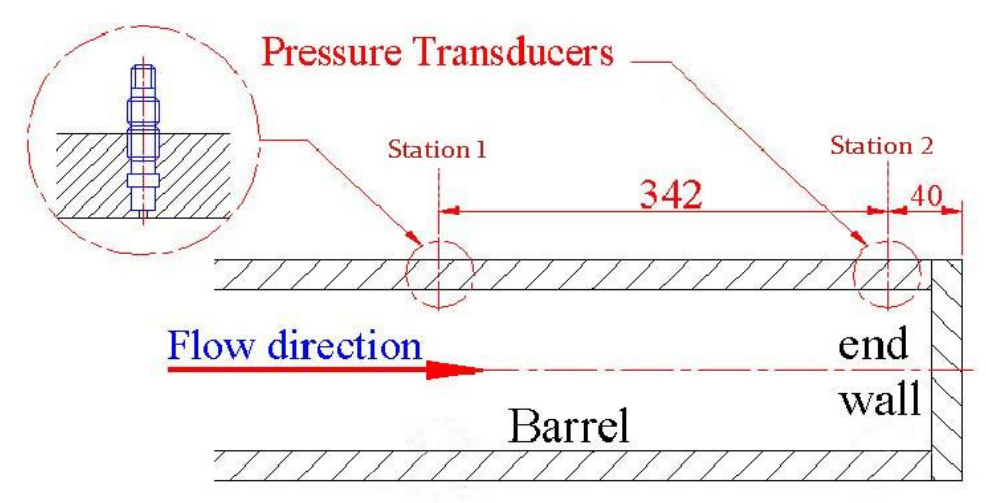

Figure 5. The pressure transducers mounted at the end of the driven section of UNITEN's high speed flow test facility.

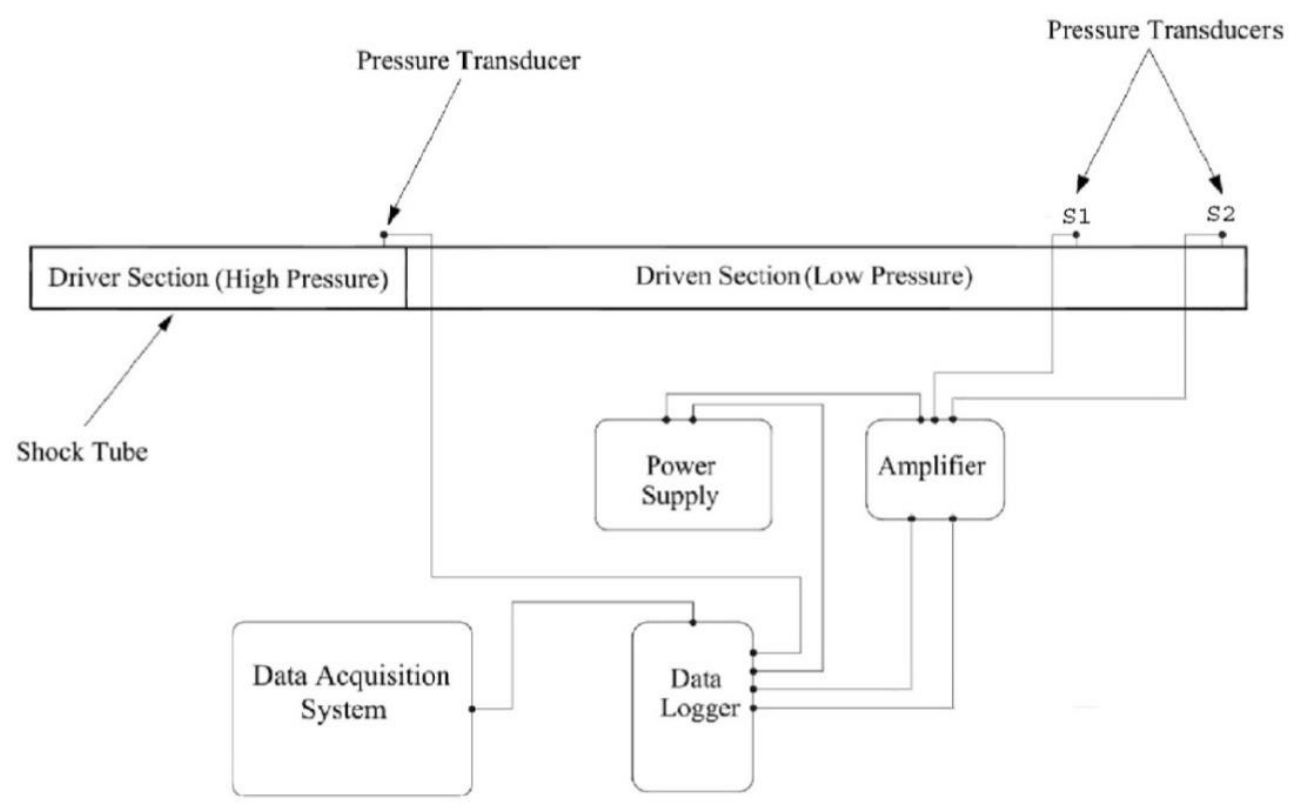

Figure 6. Data acquisition system.

Another static pressure transducer is mounted on the driver section, near to the primary diaphragm, to record the exact value of the driver pressure $\mathrm{P}_{4}$, at which the diaphragm ruptures. These three pressure transducers connect to a data acquisition system, which captures the shock wave and records the instantaneous pressure history. Figure 6 shows a schematic of the data acquisition system. This system comprises a GRAPHETIC DAQ (model midi LOGGER dual GL500) 24 channel, digital recorder with a maximum sample rate of up to $500 \mathrm{Msampling} / \mathrm{sec}$ per channel, an amplifier and a data logger. The amplifier is necessary to amplify the very small signal received from the pressure transducers and the data logger connects to a computer equipped with suitable software to record and plot the instantaneous pressure history. 


\section{RESULTS AND DISCUSSION}

The following sections present the results obtained from the experimental measurements. These results are analysed to determine the effects of the area contraction (the presence of the bush) on the peak pressure, the shock wave strength and the shock wave speed.

\section{Peak Pressure and Shock Wave Strength without Area Contraction}

Figure 7 shows the instantaneous pressure history at station (1) for the case of no area contraction, at three different diaphragm pressure ratios of 10,15 and 20. This figure clearly shows the physics of the flow inside the shock tube. Considering the pressure history at diaphragm pressure ratio $\mathrm{P}_{4} / \mathrm{P}_{1}$ of 20 , the first pressure jump represents the shock wave passing station (1), which causes the static pressure to increase instantaneously from $100 \mathrm{kPa}$ to around $360 \mathrm{kPa}$. As the shock wave proceeds to the end of the tube, it reflects and moves back in the opposite direction. The passage of the reflected shockwave at station (1) further increases the static pressure to about $980 \mathrm{kPa}$. The shock wave then interacts with the contact surface, which is following the shock wave and due to this interaction between the shock wave and the contact surface, the pressure increases further until it reaches its peak pressure value of $1450 \mathrm{kPa}$. Comparing the results at different pressure ratios, the results also clearly indicate that the peak pressure and shockwave strength increase as the diaphragm pressure ratio increases.

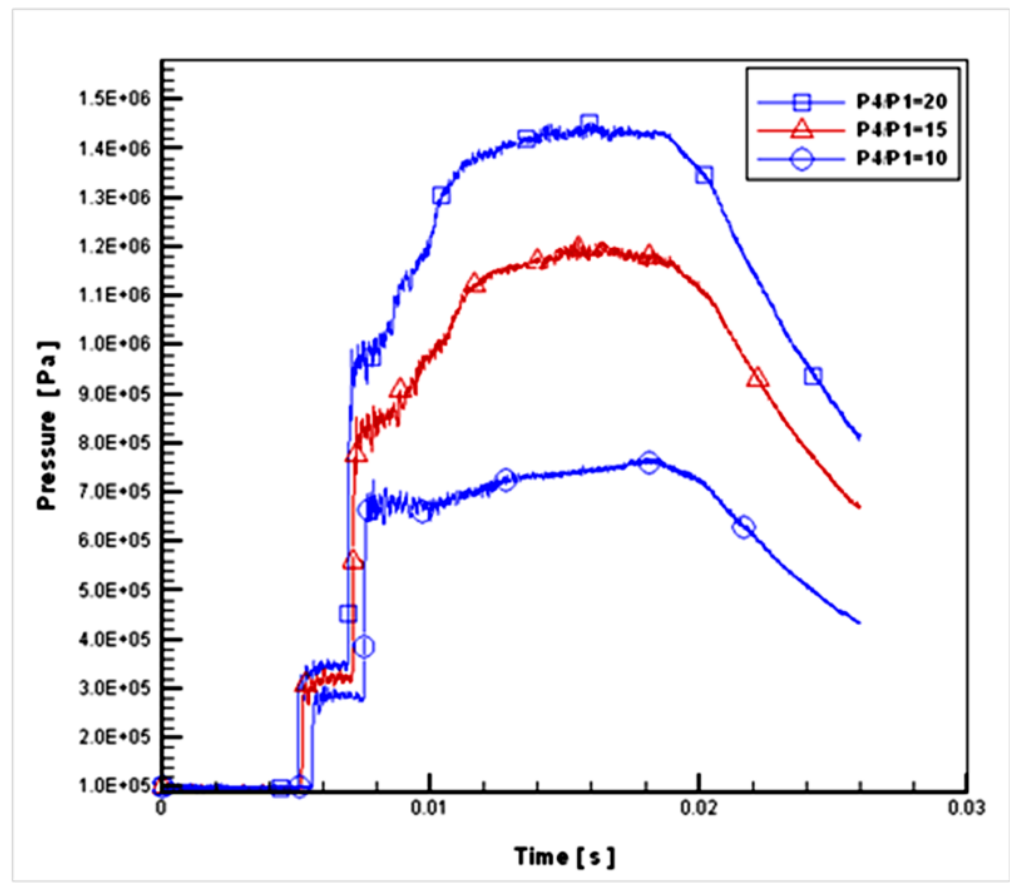

Figure 7: Pressure history at station (1) with no area contraction (air-air)

\section{Peak Pressure and Shock Strength with Area Contraction}

The same instantaneous pressure history trends occur for cases with area contraction, as shown in Figure 8. This figure shows the instantaneous pressure history at station (1) 
with the bush placed in the diaphragm section. At diaphragm pressure ratios $\mathrm{P}_{4} / \mathrm{P}_{1}$ of 20 , the recorded values of shock strength, reflected shock strength and peak pressure are $300 \mathrm{kPa}, 760 \mathrm{kPa}$, and $1000 \mathrm{kPa}$, respectively. These values are significantly below those obtained with no area contraction.

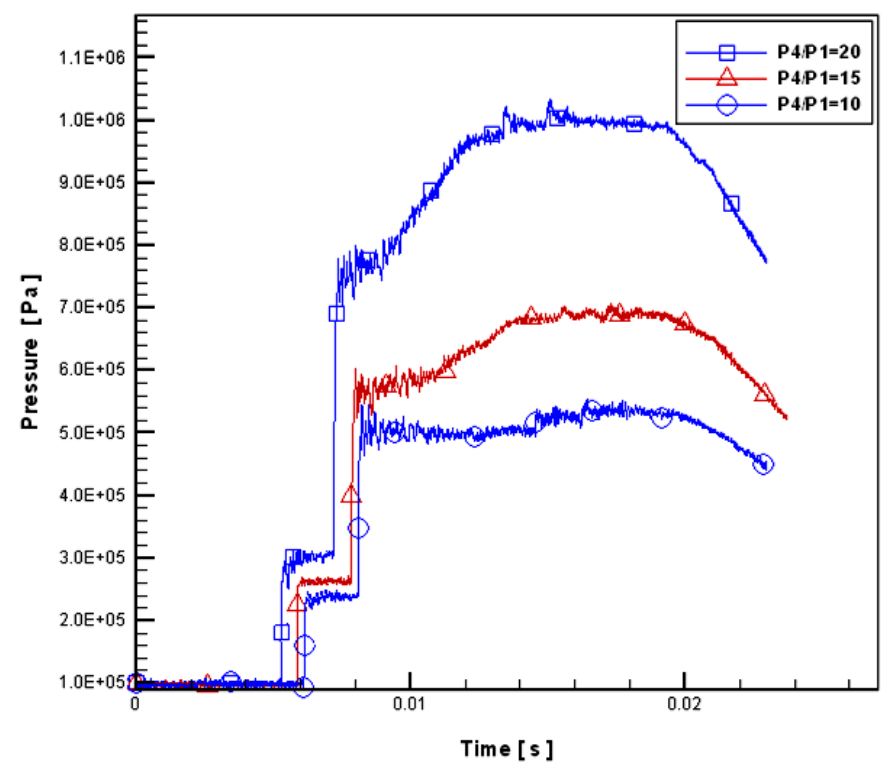

Figure 8. Pressure history at station (1) with area contraction (air-air)

For clarification, Figure 9 presents in one graph the two cases, with and without area contraction for a diaphragm pressure ratio of $\mathrm{P}_{4} / \mathrm{P}_{1}$ of 10 . The differences in the instantaneous pressure history of the two cases are obvious. The values of shock strength and peak pressure with no area contraction are $290 \mathrm{kPa}$ and $770 \mathrm{kPa}$, while these values drop to $240 \mathrm{kPa}$ and $540 \mathrm{kPa}$ for the experiment performed with area contraction. The same observations are made when diaphragm pressure ratios of 15 and 20 are used.

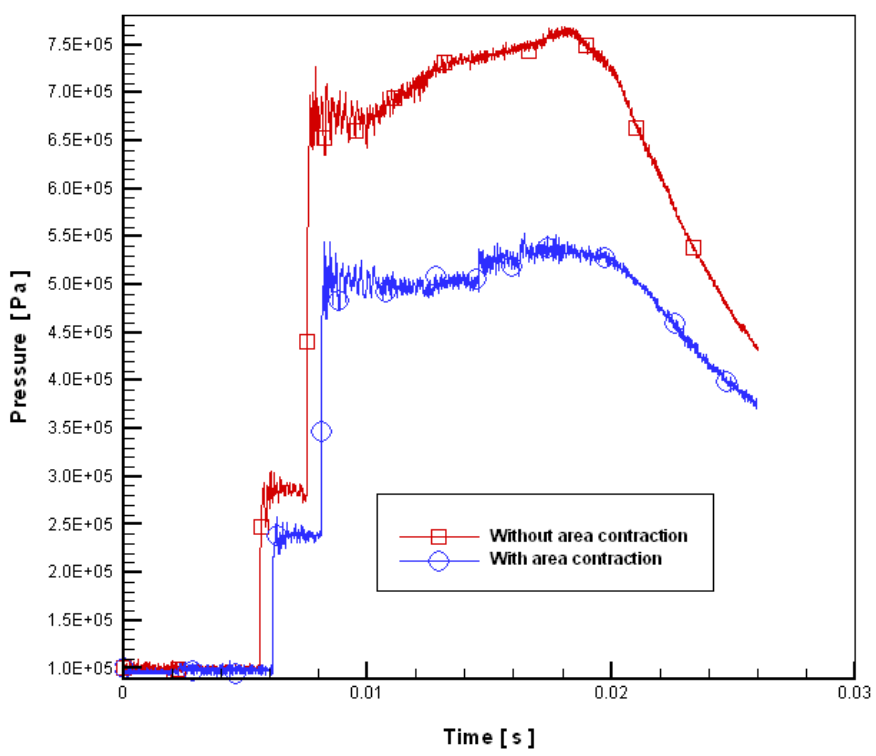

Figure 9. Pressure history at station (1) (air-air) $\left(\mathrm{P}_{4} / \mathrm{P}_{1}=10\right)$. 


\section{Shock Wave Speed}

The shock wave speed is determined from the experimental data. The distance between the two stations is known $(0.342 \mathrm{~m})$ and the time taken for the shock to travel from station 1 to station 2 can be obtained from the pressure history graph, as shown in Figure 10. This figure is a close up view of the incident shock wave region with a diaphragm pressure ratio of 10. Figure 10(a) shows that the incident shock wave with no area contraction takes $0.000624 \mathrm{sec}$ to travel from station (1) to station (2), which means that the incident shock wave speed is $548 \mathrm{~m} / \mathrm{s}$. When the bush is present in the facility (with area contraction) for the same pressure ratio, Figure 10(b) shows that the generated incident shock wave takes $0.000678 \mathrm{sec}$ to travel from station (1) to station (2). Thus, the shock wave speed is $504 \mathrm{~m} / \mathrm{s}$, which is evidently lower than that determined with no area contraction. The same observation is made when different diaphragm pressure ratios of 15 and 20 were applied.

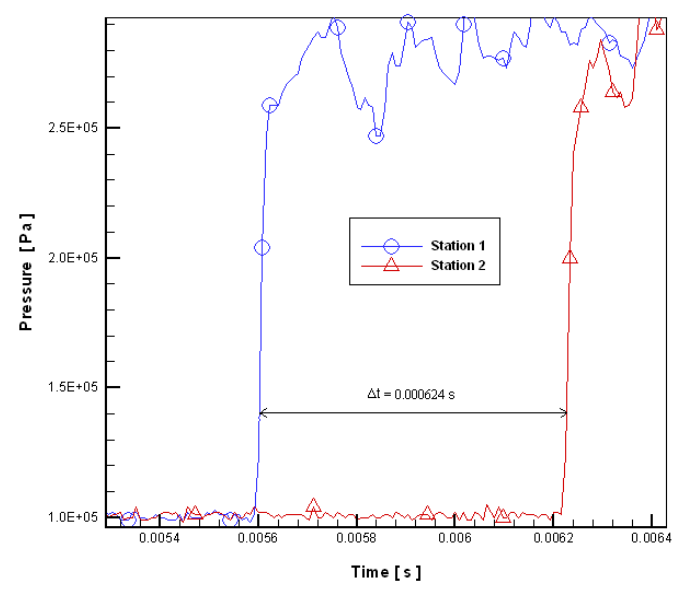

(a) Without area contraction

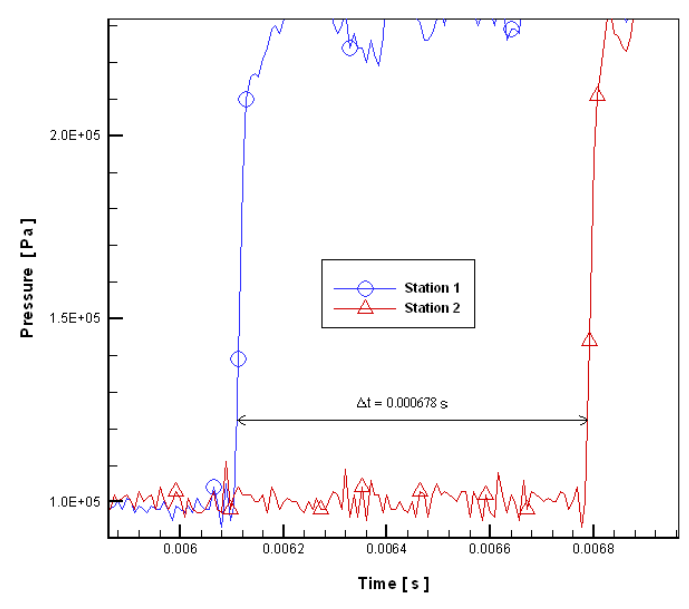

(b) With area contraction

Figure 10. The incident shock wave for diaphragm pressure ratio of 10 (Air-Air).

\section{CONCLUSION}

In this paper, the effects of area contraction on the peak pressure, shock wave strength and shock wave speed, which are important parameters determining the performance of a high speed flow test facility, have been investigated experimentally. The results show that area contraction, through placing a bush adjacent to the diaphragm in the driven section, significantly reduces peak pressure, shock wave strength and speed. At a diaphragm pressure ratio of 10 , the results reveal that the shock strength decreases by $18 \%$, the peak pressure decreases by $30 \%$ and the shock speed reduces by $8 \%$, when the shock tube is used with area contraction. Similar results were observed when using three different diaphragm pressure ratios to repeat the experiments. 


\section{REFERENCES}

Amir, A.F., Yusoff, M.Z. and Yusaf, T. 2008. Development of a short duration hypersonic test facility at Universiti Tenaga Nasional. Journal of the Institution of Engineers, Malaysia, 69(1): 19-25.

Amir, A.F., Yusoff, M.Z. and Yusaf, T. 2007. Numerical and experimental study to evaluate the performance of Universiti Tenaga Nasional short duration hypersonic test facility. In AFMC: 16th Australasian Fluid Mechanics Conference, Crown Plaza, Gold Coast, Australia, pp. 104-111.

Amir, A.F., Yusoff, M.Z. and Yusaf, T. 2008. An experimental evaluation of shock strength and peak pressure in a conventional shock tube and a free piston compressor. In IMECE2008: ASME International Mechanical Engineering Congress and Exposition, Boston, USA, pp. 1-8.

Amir, A.F., Yusoff, M.Z., Ahmed, D.I. and Shuaib, N.H. 2009. Experimental performance evaluation of short duration high speed flow shock tunnel. Proceedings of ICEE 2009 3rd International Conference on Energy and Environment, Malacca, Malaysia, pp. 379 - 384.

Aso, S., Hakim, A.N., Miyamoto, S. Inoue, K. and Tani, Y. 2005. Fundamental study of supersonic combustion in pure airflow with use of shock tunnel. Acta Astronautica 57(2-8): 384-389.

Briassulis, G., Agui, J.H., Andreopoulos, J. and Watkins, C.B. 1996. A shock tube research facility for high-resolution measurements of compressible turbulence. Experimental Thermal and Fluid Science, 13(4): 430-446.

Kaneko, M., Men'shov, I. and Nakamura, Y. 2005. Numerical simulation of nonequilibrium flow in high-enthalpy shock tunnel. Energy, 30(2-4): 165-179.

Lu, F.K., Wilson, D.R. and Matsumoto, J. 2009. Rapid valve opening technique for supersonic blowdown tunnel. Experimental Thermal and Fluid Science, 33(3): 551-554.

Sahoo, N., Mahapatra, D.R., Jagadeesh, G., Gopalakrishnan, S. and Reddy, K.P.J. 2007. Design and analysis of an accelerometer-based force balance system for shock tunnel testing. Measurement, 40(1): 93-106.

Wintenberger, E. 2002. 6 Inch shock tube experiments. 315 Guggenheim. 